\title{
Multiplicity of soliton transformations in the vicinity of the boundaries of their existence
}

\author{
W. Chang ${ }^{a}$, J. M. Soto-Crespo ${ }^{b}$, A. Ankiewicz ${ }^{a}$ and N. Akhmediev ${ }^{a}$ \\ ${ }^{a}$ Optical Sciences Group, Research School of Physical Sciences and Engineering, \\ The Australian National University, Canberra ACT 0200, Australia; \\ ${ }^{b}$ Instituto de Óptica, C.S.I.C., Serrano 121, 28006, Madrid, Spain
}

\begin{abstract}
The region of transition between solitons and fronts in dissipative systems governed by the complex GinzburgLandau equation is rich with bifurcations. We found that the number of transitions between various types of localized structures is enormous. For the first time, we have found a sequence of period-doubling bifurcations of creeping solitons and also a symmetry-breaking instability of creeping solitons. Creeping solitons may involve many frequencies in their dynamics resulting, in particular, in a variety of zig-zag motions.
\end{abstract}

Keywords: Dissipative soliton, Ginzburg-Landau equation, bifurcations

\section{INTRODUCTION}

Localized structures are widely observed in both conservative systems ${ }^{1}$ and systems far from equilibrium. ${ }^{2}$ Solitons were first found in the so-called 'integrable' systems described by the Korteweg-de Vries(KdV) equation or the nonlinear Schrödinger equation (NLSE). The latter equation can be extended to cover systems which are far from equilibrium by converting the real coefficients of the equation into complex numbers. The resulting equation is widely known as the complex Ginzburg-Landau equation (CGLE). Then NLSE can be considered as the conservative limit of the CGLE. When the coefficients are purely imaginary, we obtain the real GinzburgLandau equation as another limit. The latter has localized solutions in the form of fronts. Thus, by changing the control parameters from real to imaginary, we can have a transformation from solitons to fronts. This transformation was first studied by Hakim, Jakobsen and Pomeau ${ }^{3}$ (see also ref. 4).

This simple principle can generally be extended to more complicated systems. As an example, we consider here the higher-order complex Ginzburg-Landau equation. The addition of quintic terms makes the NLSE equation non-integrable. Soliton solutions still exist, even in this case, and a change of control parameters from real to imaginary may transform them into fronts. However, the transition is not as simple as has been suggested earlier. ${ }^{3}$ Namely, this transition consists of a series of bifurcations which occur before we enter the region of fronts. The detailed bifurcation diagram depends on the particular route that we choose in the space of control parameters. In this work, we present a particular case which has an especially complicated set of bifurcations. The route that we have chosen creates a rich variety of localized structures before it actually converges to fronts. Generally, the CGLE has many types of localized solutions. ${ }^{5}$ These can be classified as "dissipative solitons" ${ }^{6}$ What we discussing in this work is a narrow transition region in the space of parameters. This transition region is of the order of $10^{-2}$ of the value of the control parameter itself.

The model that we use is the cubic-quintic complex Ginzburg-Landau equation (CGLE). When applied to optical systems like all-optical transmission lines or passively mode-locked lasers, it can be written in the form: ${ }^{1}$

$$
i \psi_{t}+\frac{D}{2} \psi_{x x}+|\psi|^{2} \psi+\nu|\psi|^{4} \psi=i \delta \psi+i \epsilon|\psi|^{2} \psi+i \beta \psi_{x x}+i \mu|\psi|^{4} \psi,
$$

where $\psi(x, t)$ is the normalized field envelope, $x$ is the transverse variable, $t$ is the longitudinal variable, $D$ is the dispersion coefficient, $\beta$ is the spectral filtering, $\delta$ is the linear gain or loss, $\epsilon$ is the nonlinear gain, $\mu$ is the quintic gain and $\nu$ is the quintic part of the refractive index. This equation models physical systems far from equilibrium. In addition to optical systems, it can be applied to binary liquids, chemical reactions and biological systems. This sphere of knowledge is known as "the world of the complex Ginzburg-Landau equation".7

Complex Systems II, edited by Derek Abbott, Tomaso Aste, Murray Batchelor, Robert Dewar,

Tiziana Di Matteo, Tony Guttmann, Proc. of SPIE Vol. 6802, 68021D, (2008)

0277-786X/08/\$18 $\cdot$ doi: $10.1117 / 12.761199$

Proc. of SPIE Vol. 6802 68021D-1 


\section{NUMERICAL RESULTS}

We used a split-step fast Fourier transform method in order to solve Eq. (1). The parameters of the equation have been chosen around the region where the transition from a soliton solution to moving fronts occur. This region can roughly be described as a surface in the multi-dimensional space of parameters. On one side of the surface we have solitons, while on the other side they are transformed into fronts. This surface can be crossed at various points and at various angles to the surface. Thus, the route is not unique. Each specific choice creates a certain bifurcation diagram. In our case, we varied one of the system parameters $(\epsilon)$ while keeping all the others fixed. This technique is appealing due to its simplicity. Choosing any other route creates a different bifurcation diagram but does not make any conceptual difference. As the resultant parameter, we used the $L 2$-norm which is commonly known in optics as the total energy of the soliton. It is denoted here by $Q$.

The dependence of $Q$ on $\epsilon$ (i.e. the energy bifurcation diagram) is shown in Figure 1 .

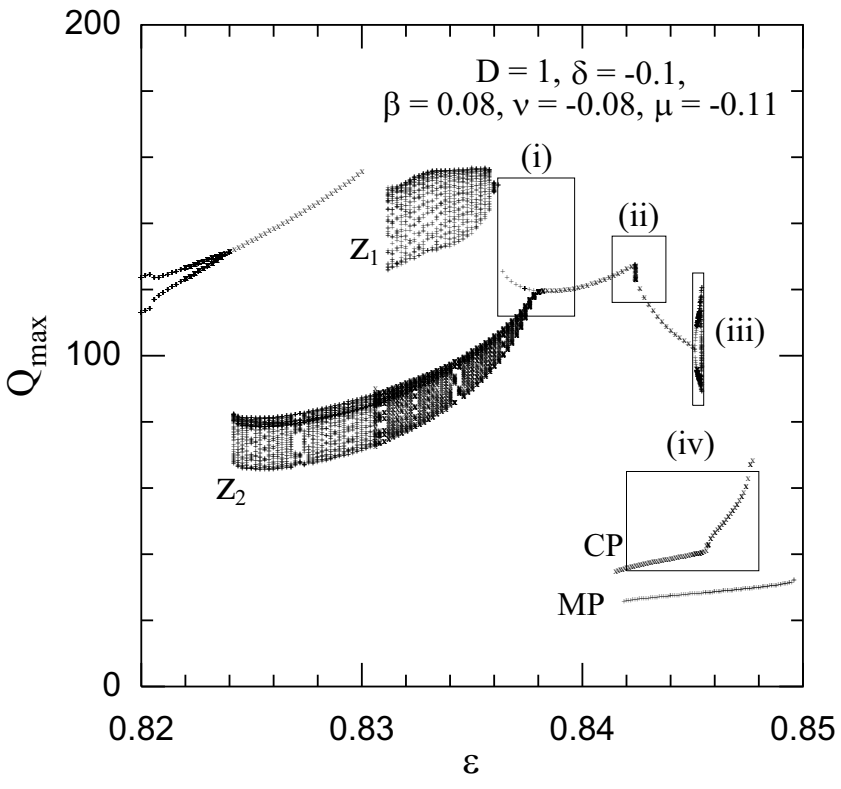

Figure 1. Energy bifurcation diagram in the region of transition between solitons and fronts in dissipative systems governed by Eq. (1). A very rich bifurcation structure is observed when $\epsilon$ is varied from 0.82 to 0.85 . Other system parameters are fixed at $D=1, \delta=-0.1, \beta=0.08, \nu=-0.08$ and $\mu=-0.11$. Special bifurcation points are enclosed with rectangles and marked with Roman numerals. These are shown in more detailed diagrams below.

There are many different types of soliton solutions which exist in this region of transition. Pulsating solitons form one type. ${ }^{5}$ For a pulsating soliton, the evolution of pulse shape repeats in a periodic manner along the direction of propagation. Pulsating solitons existing in dissipative systems are qualitatively different from the so-called 'breathers' that are found in integrable systems, and they appear far from the integrable limit of Eq. (1). This limit occurs when $\beta, \delta, \epsilon, \mu$ and $\nu$ all approach zero.

The behavior of the solitons can be more complicated than simple pulsations. It can involve movements in the transverse $(x)$ direction. One example is the so-called creeping soliton. ${ }^{5,8}$ A creeping soliton repeats its pulse shape along the propagation direction in a similar manner to a pulsating one, but, by way of contrast, it has motion in the transverse direction at the same time. This transverse motion is related to the pulsating cycle in such a way that the pulse moves more quickly at a certain point in the pulsating cycle. Below, we present more intricate behaviors of these creeping solitons involving several different motions at different frequencies. 


\section{SOLITONS WITH MULTI-FREQUENCY DYNAMICS}

Two bands of solutions, labeled $Z_{1}$ and $Z_{2}$, are the regions where the creeping solitons with multiple frequency dynamics are found. Each band corresponds to a particular type of creeping solution, i.e. they are qualitatively different. There is a continuous range of the energy maximum values for both cases, because there are two or more incommensurate frequencies involved in their motions. Each frequency is responsible for a particular type of motion. Figure 2 shows propagations of both upper and lower band solutions at $\epsilon=0.834$.
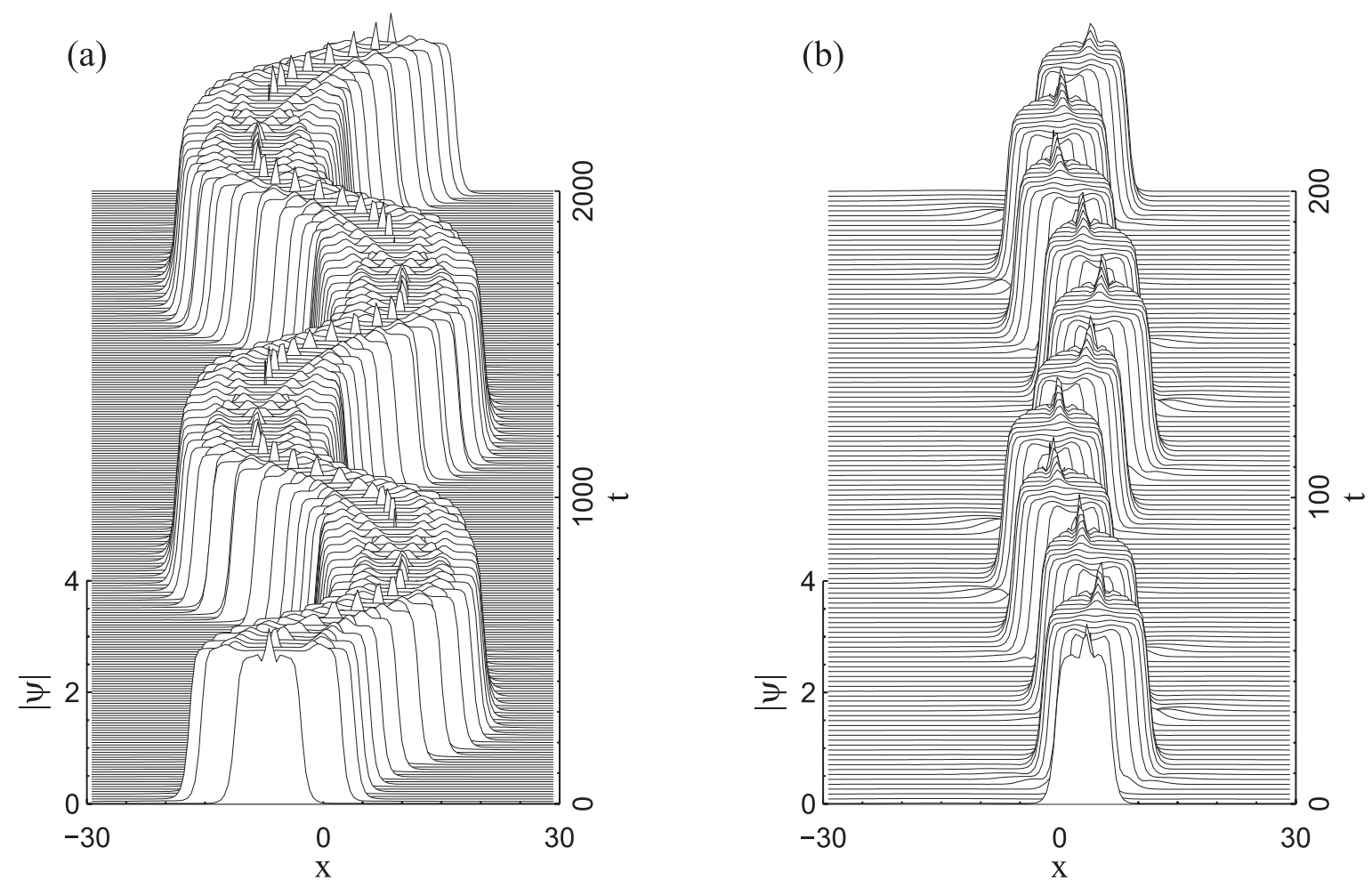

Figure 2. Pulse evolution along the propagation direction. Both diagrams are solutions of the system with its parameters fixed at $D=1, \delta=-0.1, \beta=0.08, \nu=-0.08, \mu=-0.11$ and $\epsilon=0.834$. (a) and (b) correspond to the bands of creeping solutions, $Z_{1}$ and $Z_{2}$, in Figure 1 , respectively.

The upper band has two dynamical frequency components. The higher frequency is related to the pulsation of the pulse, which also causes the pulse to 'creep'. The lower frequency component causes the pulse to move in a zig-zag motion. The graph plotted in Figure 3(a) shows clearly how these two frequencies affect the behavior of the soliton.

The lower band also has multiple frequencies governing its motion. There is a frequency component that is related to the pulsation and the corresponding creeping motion. There is also another frequency that moves the soliton in a zig-zag motion. From Figure 3(b), the zig-zag motion does not exactly repeat itself, but instead, it shifts in the $x$ direction. This is caused by another lower frequency component involved in the behavior of the soliton. The effect of this third frequency component becomes more evident if we increase the scale of this graph in $t$, as shown in Figure 4. As we can see, the third frequency component also causes the soliton to move in a zig-zag motion, but this time with a much longer period. Hence this soliton in the lower band has two zig-zag motions. 

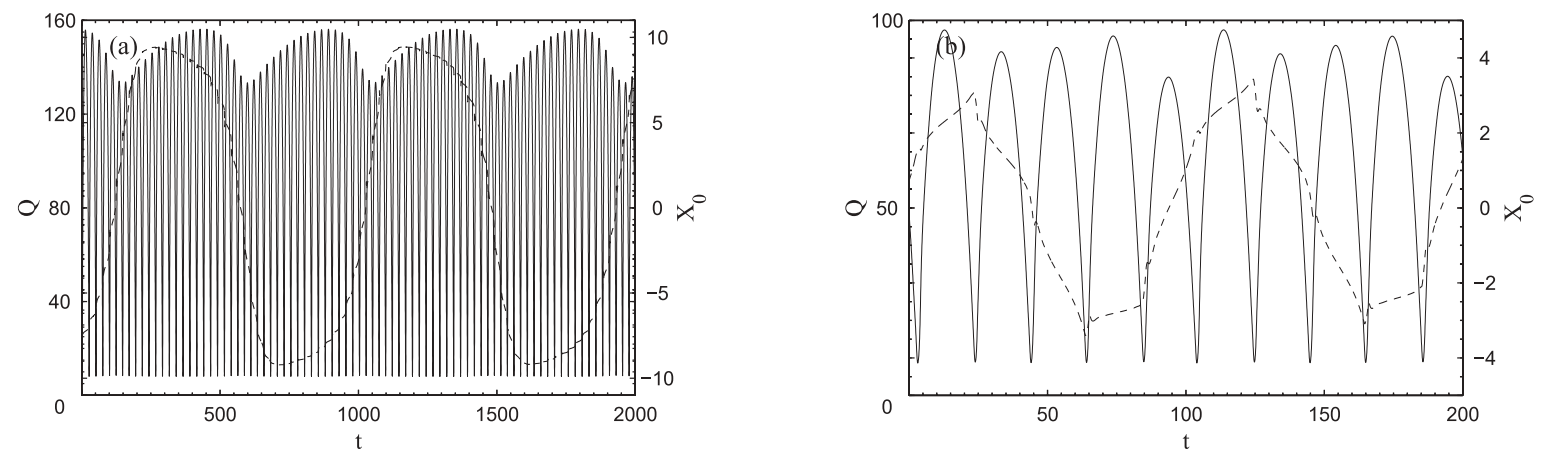

Figure 3. Evolution of the soliton energy along $t$ for the soliton propagation shown in Figure 2: (a) corresponds to the upper band $\left(Z_{1}\right)$ while (b) corresponds to the lower band $\left(Z_{2}\right)$. The center-of-mass (first momentum), $X_{0}$, for each case is also presented and is depicted with dashed lines.
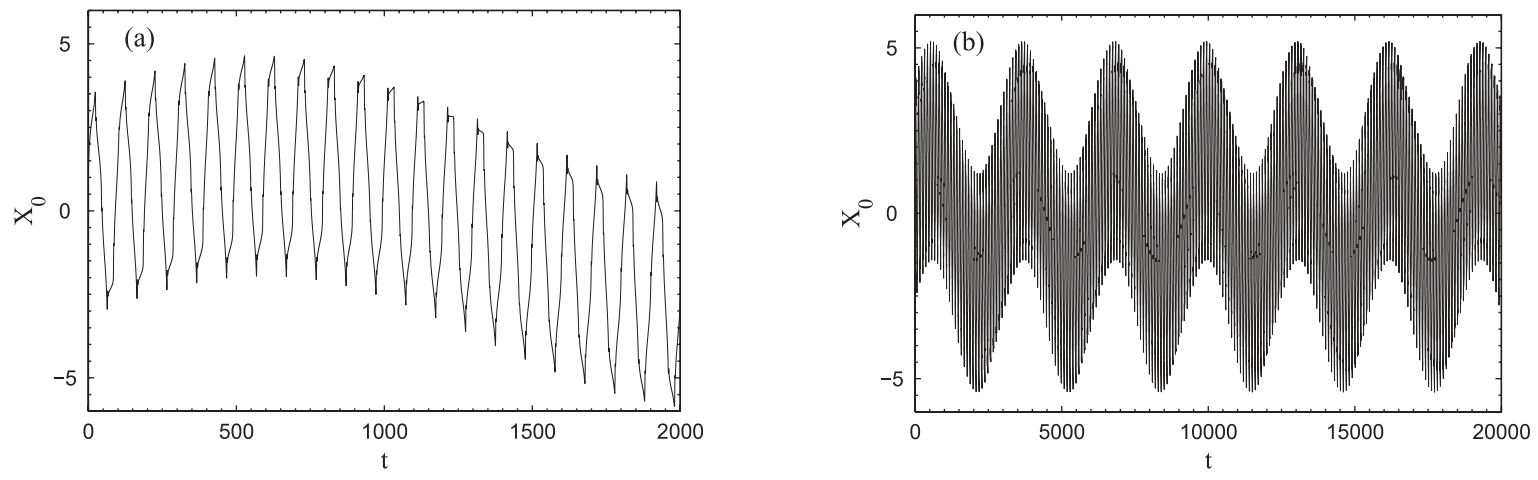

Figure 4. Position of the soliton's center-of-mass, $X_{0}$, plotted along its propagation direction for the lower band $\left(Z_{2}\right)$ with two different scales; (b) clearly shows that there are three frequency components in its motion.

\section{SYMMETRY-BREAKING BIFURCATIONS}

A pitch-fork bifurcation indicates broken symmetry. CGLE is symmetric relative to the change of sign of $x$. However, solutions don't have to be symmetric. Thus, there may be transformations from symmetric solution to an asymmetric at certain values of parameters. There are two types of symmetry-breaking bifurcations in the theory of bifurcations, viz. sub-critical and super-critical. We have observed both of these types in our numerical simulations. Each one involves a bifurcation of a symmetric limit-cycle to two asymmetric limitcycles. Clearly, bifurcations of limit cycles are distinct from typical cases of symmetry-breaking involving fixed points. A bifurcation from a stationary soliton to a moving pulse has been found in ref. 9 for a case where the cubic Ginzburg-Landau equation is coupled to another equation. This system represents two coupled nonlinear optical fibres, where one fibre also features dispersion. The authors of ref. 9 find a sub-critical symmetry-breaking bifurcation which transforms a stationary pulse into a moving one. However, for the range of parameters studied here, the moving pulse branch always has non-zero velocity, and we do not observe such a bifurcation for the given set of parameters.

\subsection{Sub-critical symmetry-breaking}

A sub-critical symmetry-breaking bifurcation is observed in Region (i) of Figure 1. This region is magnified and plotted in Figure 5. This type of bifurcation creates a small region where two stable branches of solutions co-exist (hysteresis). In an earlier work,${ }^{9}$ hysteresis has been found in the transition from moving pulse solutions to moving hole solutions of the CGLE. This occurred over a small range of the parameter being varied, as in our case. 
Figure 5(b) shows that the transition from one branch to the other branch depends on the direction in which $\epsilon$ is varied. When $\epsilon$ is increased, the transition from the creeping branch to the pulsating branch follows the down arrow on the right-hand-side, whereas if $\epsilon$ is decreased, the transition occurs at a slightly lower value of $\epsilon$, following the up arrow on the left-hand-side.
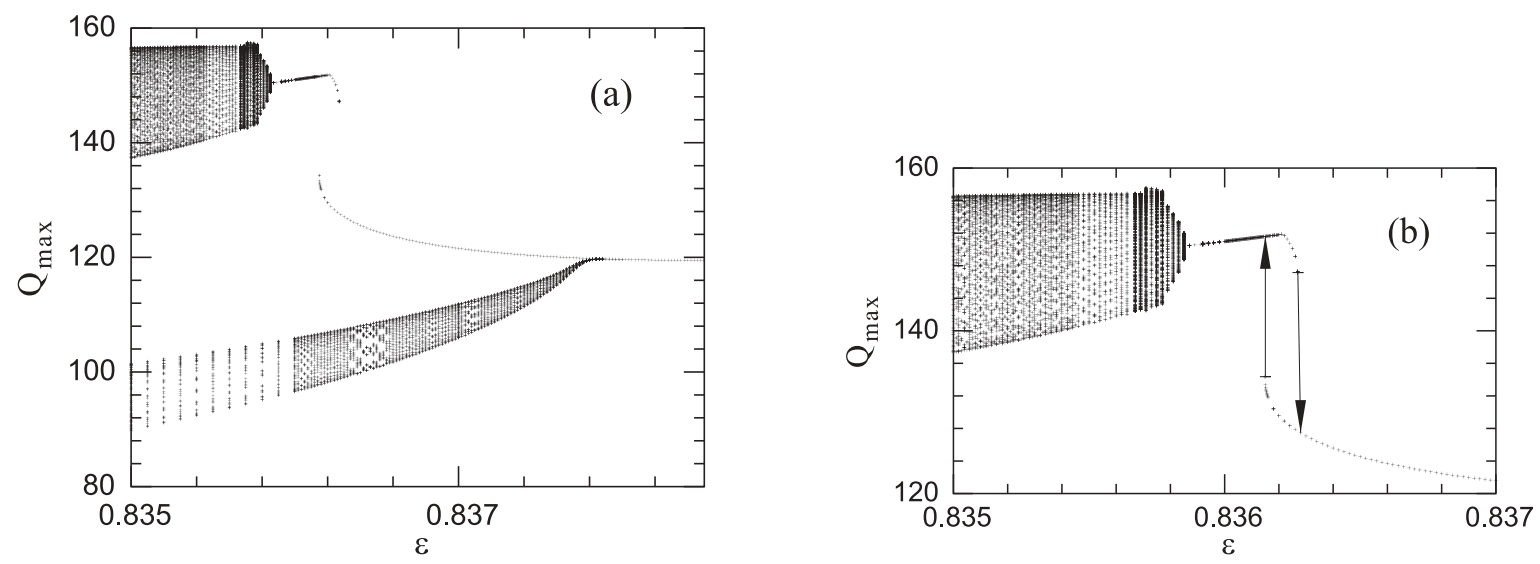

Figure 5. Region (i) of Figure 1. Bistability of a creeping soliton and a pulsating soliton is observed in (b). This is a sub-critical symmetry-breaking bifurcation.

\subsection{Super-critical symmetry-breaking}

Another symmetry-breaking bifurcation is found at the end of the pulsating branch near $\epsilon=0.8425$ (Region (ii) of Figure 1). At this point, there is a bifurcation from a pulsating soliton to a creeping soliton. With a careful numerical study around this point, we have found that there is an unstable branch of pulsating solutions that continues from the stable branch of the left-hand-side. This unstable branch is shown with a dashed line in Figure $6(a)$. In the region on the right-hand-side of the bifurcation point, even if we have started off with a symmetric initial condition, this function first converges to a pulsating solution, then the solution is transformed into a creeping soliton after a limited propagation distance, as shown in Figure 6(b). This suggests that symmetric solutions are unstable beyond the bifurcation point. The creeping branch on the right-hand-side is degenerate, since it represents two separate creeping branches, where the creeping movements are in opposite directions.

\section{OTHER BIFURCATIONS}

There are other bifurcations that are also of interest in this work. At the end of the creeping solution branch that has been mentioned in Section 4.2, the creeping solution branch follows a sequence of period doubling bifurcations. A magnified diagram of Region (iii) of Figure 1 suggests that these period-doubling bifurcations will end up following the route to chaotic behavior. Due to the creeping nature of this branch, the chaotic solitons that appear at the end are still moving localized solutions. The velocities of these solitons in the transverse direction vary chaotically along the propagation, but the motion is only in one direction, and this is determined by the perturbation at the beginning. This branch ends near $\epsilon=0.84543$ when the period of pulsation increases without limit and the soliton disappears to form two moving fronts. At this point, we cross the surface of transition from solitons to fronts.

The simultaneous existence of two or several types of stable localized solutions is one of the features of the system governed by the CGLE. There are two more branches of solutions that co-exist with the solutions that we have discussed in the previous sections. One is the branch labeled 'CP' (composite pulse). A composite pulse is a stationary solution and it has two fronts located symmetrically on the sides of the pulse, and a small bump in the middle. Following this branch in the direction of increased $\epsilon$ in Figure 1, the slope of the branch changes rapidly near $\epsilon=0.8454$ in Region (iv), and this is another point of bifurcation. The stationary soliton 


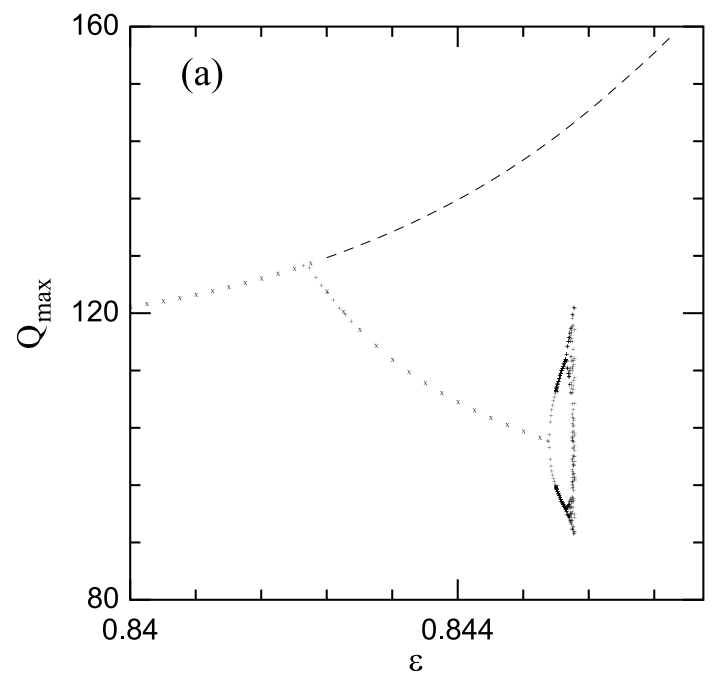

(b)

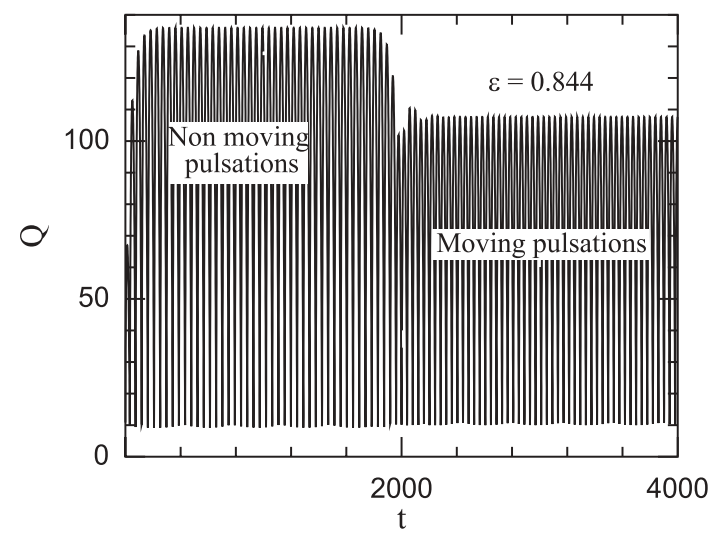

Figure 6. (a) Region (ii) of Figure 1. Super-critical symmetry-breaking bifurcation. The unstable branch of the pulsating solution is shown by the dashed line; (b) After some propagation distance, a pulsating solution on the unstable branch transforms into a creeping soliton with a lower maximum energy.

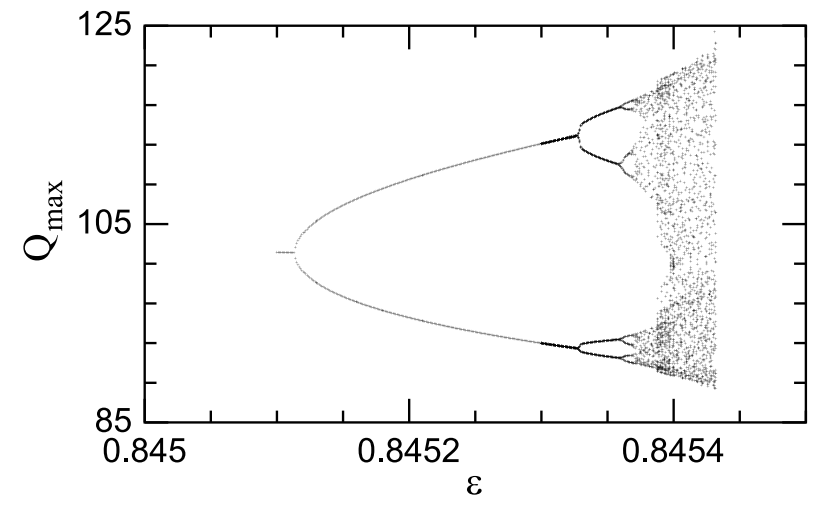

Figure 7. Region (iii) of Figure 1. A period doubling and quadrupling sequence to chaos for a creeping solution branch.

starts to pulsate beyond this point, as shown by the split of the energy curve in Figure 8 . The two dotted curves correspond to the minimum and maximum values of oscillating energy. Such a transition from a fixed point solution to a limit-cycle is classified as an Andronov-Hopf bifurcation. We stress here that in contrast to the results from standard nonlinear dynamics this bifurcation occurs in the infinite dimensional system.

The lowest branch, labeled 'MP' (moving pulse), is the other solution that exists simultaneously with the solutions mentioned above. This solution does not change its asymmetric shape, but has a fixed velocity in the transverse direction while it is propagating, ${ }^{11}$ where the direction of this transverse motion is dependent on the initial condition.

At the end of this branch, two fronts of the pulse move away from each other, and the soliton disappears. Again, this transition corresponds to crossing the boundary between solitons and fronts. Similar transitions can be observed when we cross this boundary at other points of this surface in the space of parameters. The sequence of bifurcations may be different, of course. Nevertheless, it is hard to imagine that the bifurcation diagram would be any simpler than the one we presented in this work. 


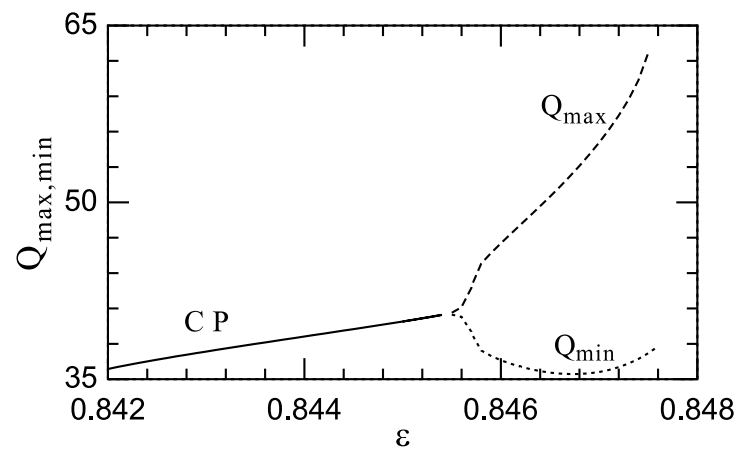

Figure 8. Region (iv) of Figure 1. This shows the birth of a limit cycle from a fixed point i.e. an Andronov-Hopf bifurcation.

\section{CONCLUSIONS}

In conclusion, we have studied the transformations of localized structures, from solitons to fronts, which are observed for the complex cubic-quintic Ginzburg-Landau equation when the control parameters are changed. We have found that a relatively narrow region of the parameter space is rich with bifurcations. We have described most of them here and have presented the detailed bifurcation diagram.

\section{ACKNOWLEDGMENTS}

This research was supported under Australian Research Council's Discovery Projects funding scheme (project number DP0663216). The work of J.M.S.C. was supported by the M.E.y C. under contract FIS2006-03376.

\section{REFERENCES}

1. N. Akhmediev and A. Ankiewicz, Solitons, nonlinear pulses and beams, Chapman and Hall, London, 1997.

2. S. Fauve and O. Thual, "Solitary waves generated by subcritical instabilities in dissipative systems," Phys. Rev. Lett. 64, p. 282, 1990.

3. V. Hakim, P. Jakobsen, and Y. Pomeau, "Fronts vs solitary waves in nonequilibrium systems," Europhysics Letters 11, pp. 19-24, 1990.

4. B. A. Malomed and A. A. Nepomnyashchy, "Kinks and solitons in the generalized Ginzburg-Landau equation," Phys. Rev. A 42, p. 6009, 1990.

5. N. Akhmediev, J. M. Soto-Crespo, and G. Town, "Pulsating solitons, chaotic solitons, period doubling, and pulse coexistence in mode-locked lasers: CGLE approach," Phys. Rev. E 63, p. 056602, 2001.

6. N. Akhmediev and A. Ankiewicz, Dissipative solitons, ch. Dissipative solitons in the complex GinzburgLandau and Swift-Hohenberg equations. Springer-Verlag, Berlin, 2005.

7. I. S. Aranson and L. Kramer, "The world of the complex Ginzburg-Landau equation," Rev. Mod. Phys. 74, p. 118, 2002.

8. W. Chang, A. Ankiewicz, and N. Akhmediev, "Creeping solitons of the complex Ginzburg-Landau equation," Phys. Lett. A 362, pp. 31-36, 2007.

9. O. Descalzi, H. R. Brand, and J. Cisternas, "Hysteretic behavior of stable solutions at the onset of a weakly inverted instability," Physica A 371, pp. 41-45, 2006.

10. H. Sakaguchi and B. A. Malomed, "Instabilities and splitting of pulses in coupled Ginzburg-Landau equations," Physica D 154, pp. 229-239, 2001.

11. V. V. Afanasjev, N. N. Akhmediev and J. M. Soto-Crespo, "Three forms of localized solutions of the quintic complex Ginzburg-Landau equation,” Phys. Rev. E 53, p. 1931, 1996. 